\title{
G\{ ISKO
}

\section{PREPRINT}

\section{La descripción archivística y la curaduría digital: relaciones y perspectivas.}

Dunia LLanes Padrón ${ }^{1}$, Manuela Moro Cabero ${ }^{2}$

${ }^{1}$ http://orcid.org/0000-0001-8639-4706, Universidad de La Habana, Departamento de Ciencias de la Información, La Habana, Cuba, duniallp@yahoo.es

2 http://orcid.org/0000-0001-5301-1924, Universidad de Salamanca, Departamento de Biblioteconomía y Documentación, Salamanca, España, moroca@usal.es

Tipo de contribución: comunicación 


\section{Resumen:}

El siglo XXI se caracteriza por el impulso de la economía digital y de las tecnologías basadas en Internet. En este escenario, la Archivística, influida por la transformación digital, ha evolucionado en sus concepciones teóricas y prácticas, combinando dos intereses fundamentales: la consolidación de la memoria y la gestión proactiva y distribuida de datos, información y documentos. Como consecuencia, procesos como el de descripción o de preservación se centran en recursos complejos observándose en ellos innovaciones considerables atendiendo, en primer lugar, a sus conceptualizaciones teóricas y, en segundo término, al empleo de la normalización. La descripción, el almacenamiento y conservación de datos, información y documentos han cambiado considerablemente, motivado por la incidencia directa de las tecnologías en el surgimiento de múltiples categorías de objetos digitales (OD) dotados de estructuras heterogéneas sujetas a notable obsolescencia. En este ecosistema electrónico resultan necesarias nociones sobre Curaduría Digital; este modelo comprende la descripción e información del OD, así como una planificación de la preservación de dicho OD a lo largo de su ciclo de vida. Ello conlleva acciones diversas, tales como la validación del OD, asignación de metadatos de preservación y de representación del recurso digital. En este modelo la descripción, (asignación de metadatos administrativos, descriptivos, técnicos, contextuales, estructurales y de preservación), la representación para su descubrimiento y la preservación se establecen como dimensiones centrales. Este estudio tiene como objetivo delimitar las relaciones que existen entre la descripción archivística y algunas dimensiones del ciclo de vida de la Curaduría Digital. Con este fin, se analiza el potencial que representan los nuevos Modelos Conceptuales de Descripción Archivística (MCDA), exponiéndose cómo estos pueden contribuir en el proceso permanente de descripción y preservación de objetos digitales. Asimismo, se analiza el ciclo de vida de la $\mathrm{CD}$, con especial atención en las dimensiones de descripción, acceso y reutilización de información. El estudio es fundamentalmente de naturaleza cualitativa y de tipo exploratorio. Los entornos electrónicos exigen demostraciones de relaciones e interoperabilidad de la información ante sus diversos sistemas de gestión; por tanto, las normas técnicas, los modelos conceptuales, los desarrollos ontológicos -elaborados en el seno de ISO, del ICA, así como de otros organismos nacionales-, aglutinados demuestran gran utilidad. A los archivos, en calidad de mediadores entre productores y usuarios necesitados de información y/o en calidad de centros de investigación y de cultura, les corresponde ofrecer inteligibilidad de la información, facilitar su uso y su reutilización, además de conservar los recursos digitales.

Palabras clave: descripción archivística; representación de información; modelos conceptuales; curaduría digital; reutilización de información, interoperabilidad semántica. 


\section{Introducción}

El siglo XXI se caracteriza por el impulso de la economía digital, de las tecnologías basadas en Internet y por su implementación en heterogéneos contextos de producción, administración, culturales y de servicios, entre otros. En este escenario, la Archivística, mediada por la transformación digital, ha evolucionado en sus nociones teóricas y prácticas, combinando dos intereses fundamentales: la consolidación de la memoria y la gestión proactiva y distribuida para facilitar el acceso, uso, preservación e interoperabilidad de datos, información y documentos.

Las funciones se adaptan a los cambios provocados por el nuevo modelo tecnológico, los cuales inciden en el objeto, la finalidad y los agentes implicados, sean estos productores y/o usuarios. El objeto de trabajo de los archivos -recurso digital- se presenta heterogéneo y voluminoso, mientras que productores y agentes emplean las tecnologías tanto para su producción como para su consulta y explotación de dicho recurso. Como consecuencia, procesos tales como el de descripción o de preservación se centran en recursos complejos (geoespaciales, audiovisuales, derivados de redes sociales, entornos web, entornos de office, etc.), observándose en ellos innovaciones considerables atendiendo, en primer lugar, a sus conceptualizaciones teóricas y, en segundo término, al empleo de la normalización.

En el caso del proceso de descripción se han actualizado sus concepciones y prácticas; en el presente, se fundamenta en la representación exacta de varias entidades archivísticas tomando como guía esquemas normalizados. Se facilita de este modo la interrelación y la integración de los contenidos archivísticos en Internet. Para llegar a este punto fue y sigue siendo determinante el proceso normalizador desarrollado, el cual se ha focalizado con un alcance internacional o/y nacional, atendiendo a la procedencia y uso de los modelos creados.

Asimismo, el almacenamiento y conservación de datos, información y documentos han cambiado considerablemente, desde sus preceptos hasta sus prácticas; motivado por la incidencia directa de las tecnologías en el surgimiento de múltiples categorías de objetos digitales (en adelante OD) dotados de estructuras heterogéneas (formatos) sujetas a notable obsolescencia. Ante estas contingencias, resultan insuficientes los métodos procedentes del entorno analógico para preservar los objetos creados en ambientes digitales (born digital). La preservación (voz “conservación”) es definida en el entorno UNE-ISO (AENOR 2011, p.16) como "el conjunto de procesos y operaciones realizados para garantizar el mantenimiento de los documentos a lo largo del tiempo". En esta línea, la Guía de aplicación de la norma técnica de interoperabilidad de políticas de gestión de documentos electrónicos (DTIC, 2016, p.30) ahonda en la necesidad de adoptar nuevas estrategias de preservación digital con los siguientes objetivos: 1- Evitar pérdida o degradación de las características; 2- Implementar procedimientos para garantizar el mantenimiento de contenido, contexto, componentes del OD y su valor probatorio; y 3-Mantenerlo a lo largo del tiempo. Lógicamente, se han producido cambios que afectan no sólo al objeto de trabajo, sino que también a los objetivos, la finalidad operativa y tiempo de actuación. Mientras que la conservación tradicional se ha centrado en el objeto físico para garantizar su uso, la digital lo hace en el aseguramiento de datos e información contenidos en el recurso digital, de modo 
fidedigno, auténtico e íntegro, así como en su disponibilidad para el acceso, comprensibilidad, uso y reutilización. Además, debe considerar el despliegue de cualquier acción necesaria para el logro de estos objetivos. De este modo, se ha evolucionado desde una conservación benigna (focalizada en aspectos medioambientales y de manipulación), esporádica y de acciones puntuales, a una preservación activa, dinámica y continuada que precisa acometerse durante todo el ciclo de vida del recurso digital. En este sentido, la normalización ha contribuido al establecimiento de principios básicos y técnicas para afrontar estrategias de preservación con el fin de garantizar el acceso, uso, reutilización de la memoria mundial.

Por consiguiente, en este ecosistema electrónico resultan necesarias nociones sobre Curaduría Digital (CD); este modelo requiere un análisis contextual previo para una posterior implementación de las acciones que garanticen el $\mathrm{OD}$, considerando los resultados de dicho análisis. En CD, esta investigación responde al término de “conceptualización”. Comprende por lo tanto, la descripción e información del OD, así como una planificación de la preservación de dicho OD a lo largo de su ciclo de vida. Ello conlleva acciones diversas, tales como la validación del OD, de asignación de metadatos de preservación y de la información de representación del recurso digital, de garantía sobre una estructura autorizada y aceptable para el OD, etc. Todas estas acciones se encaminan a garantizar que los recursos de información digital sean auténticos, confiables, íntegros y se mantengan disponibles (accesibles, usables y reutilizables, si fuera el caso) desde su creación/recepción o captura. En este modelo la descripción, (asignación de metadatos administrativos, descriptivos, técnicos, contextuales, estructurales y de preservación), la representación para su descubrimiento y la preservación se establecen como dimensiones centrales.

Este estudio, con la finalidad de aportar una respuesta, incluye la formulación del objetivo de delimitar las relaciones que existen entre la descripción archivística y algunas dimensiones del ciclo de vida de la Curaduría Digital. Con este fin, se analiza el potencial que representan los nuevos Modelos Conceptuales de Descripción Archivística (MCDA), exponiéndose cómo estos pueden contribuir en el proceso permanente de descripción de objetos digitales. Asimismo, se analiza el ciclo de vida de la $\mathrm{CD}$, con especial atención en las dimensiones de descripción, acceso, uso y reutilización de información.

El estudio es fundamentalmente de naturaleza cualitativa y de tipo exploratorio. Una metodología cualitativa es apropiada para el tipo de investigación que se presenta, donde se analizan algunas particularidades de los MDCA y del modelo de Curaduría Digital. A partir de este análisis, se exponen las ventajas del uso de modelos conceptuales dentro del ciclo de vida de la CD para facilitar la accesibilidad, la gestión, la reutilización y la preservación de recursos digitales.

\section{La descripción del patrimonio archivístico a partir de modelos conceptuales}

La descripción archivística es uno de los procesos que más variaciones y adaptaciones ha tenido como resultado de las transformaciones digitales. Los cambios se dirigen a una concepción pluridimensional de la representación, abandonando las nociones unidimensionales centradas, principalmente, en el documento. El proceso de normalización ha sido determinante para las adaptaciones al nuevo esquema digital; la 
normalización se enfoca en superar el tradicional propósito técnico de descripción de contenidos basado en instrumentos de referencia diversos. Se centra en sistematizar la representación de sus contenidos y su codificación, a partir de modelos conceptuales y sus ontologías para facilitar el acceso y la interoperabilidad entre los diversos sistemas de información. Estas orientaciones manifiestan una transición paulatina en la descripción: se bascula del uso de normas de estructura de datos al uso de modelos conceptuales unificados con sus respectivos desarrollos ontológicos para la web semántica.

Todas estas mutaciones han sido sistematizadas en los Modelos Conceptuales de Descripción definidos para Archivo y publicados en los últimos 10 años. Existen en la actualidad, cinco MCDA, de carácter nacional: el Australian Government Recordkeeping Metadata Standard (2008), el Technical Specifications for the Electronic Recordkeeping Metadata Standard (2008) de Nueva Zelanda, el Modelo Conceptual de Descripción Archivística y Requisitos de Datos Básicos de las Descripciones de Documentos de Archivo, Agentes y Funciones, de España (20122017), el Finnish Conceptual Model for Archival Description (2013) y, finalmente, uno de alcance internacional conocido como Records in Contexts. A Conceptual Model for Archival Description (RiC-CM), el cual fue elaborado por el ICA, siendo etidado en 2016.

En todos estos modelos se definen entidades archivísticas, atributos de descripción y relaciones. Estos elementos perfilan las dimensiones de la descripción en la actualidad. Las entidades constituyen los objetos archivísticos que serán descritos y representados en los sistemas de información; los atributos son los metadatos (de contexto, contenido y estructura) que facilitarán la representación de contenidos. Por su parte, las relaciones son las vinculaciones que se establecen entre las entidades, a través de ellas se interrelaciona la información, lo que facilita la reutilización de datos entrelazados. Seguidamente, se aporta un ejemplo comparado sobre el desarrollo de las entidades en los diversos modelos anteriormente citados.

Tabla 1. Entidades en los MCDA

\begin{tabular}{|c|c|c|c|c|}
\hline \multicolumn{5}{|c|}{ MODELOS CONCEPTUALES DE DESCRIPCIÓN ARCHIVÍSTICA } \\
\hline \multicolumn{5}{|c|}{ ENTIDADES } \\
\hline AUSTRALIA & $\begin{array}{c}\text { NUEVA } \\
\text { ZELANDA }\end{array}$ & ESPAÑNA & FINLANDIA & $I C A$ \\
\hline \multirow[t]{4}{*}{ Documento } & \multirow[t]{4}{*}{ Documento } & \multirow[t]{4}{*}{ Documento } & $\begin{array}{l}\text { Recursos } \\
\text { Información }\end{array}$ & Documento \\
\hline & & & Expresión & Componente \\
\hline & & & Manifestación & Documental \\
\hline & & & Item & $\begin{array}{l}\text { Conjunto } \\
\text { Documental }\end{array}$ \\
\hline \multirow[t]{3}{*}{ Agente } & \multirow[t]{3}{*}{ Agente } & \multirow[t]{3}{*}{ Agente } & \multirow[t]{3}{*}{ Agente } & Agente \\
\hline & & & & $\begin{array}{l}\text { Ocupación } \\
\text { (Profesión) }\end{array}$ \\
\hline & & & & Posición \\
\hline \multirow{3}{*}{$\begin{array}{l}\text { Función } \\
\text { (Business) }\end{array}$} & \multirow{3}{*}{$\begin{array}{l}\text { Función } \\
\text { (Business) }\end{array}$} & \multirow{3}{*}{$\begin{array}{l}\text { Función y sus } \\
\text { divisiones }\end{array}$} & \multirow{3}{*}{ Función } & Función \\
\hline & & & & $\begin{array}{l}\text { Función } \\
\text { Abstracta }\end{array}$ \\
\hline & & & & Actividad \\
\hline
\end{tabular}




\begin{tabular}{l|l|l|l|l}
\hline \hline $\begin{array}{l}\text { Norma } \\
\text { Mandato) }\end{array}$ & $\begin{array}{l}\text { Norma } \\
\text { (Mandato) }\end{array}$ & Norma & $\begin{array}{l}\text { Norma } \\
\text { (Mandato) }\end{array}$ & $\begin{array}{l}\text { Norma } \\
\text { (Mandato) }\end{array}$ \\
\hline \hline Relación & Relación & - & - & - \\
\hline \hline- & - & $\begin{array}{l}\text { Concepto } \\
\text { Asunto/ } \\
\text { Acontecimiento }\end{array}$ & Materia & Concepto/Asunto \\
\hline \hline- & Lugar & Lugar & Lugar \\
\hline \hline- & - & - & Evento Temporal & Período/Fechas \\
\hline \hline- & - & - & Ciclo de Vida & - \\
\hline \hline
\end{tabular}

Fuente: Elaboración propia

En todos los modelos están presentes entidades definidas en normas de descripción tales como ISAD(G), ISAAR(CPF), ISDIAH e ISDF, unificando todos los componentes del conjunto normativo. Asimismo, se puede apreciar en los MCDA elementos coincidentes con las FRBR (Requisitos Funcionales para Registros Bibliográficos) y el CRM (Conceptual Reference Model) para Museos. Las entidades documento, agente, función, norma, lugar, período y asunto son denominadores comunes en todo los modelos conceptuales desarrollados en las Ciencias de la Información y constituyen entidades principales. Por ende, se subraya la posibilidad de creación de un modelo semántico único que facilite la representación, la relación y la interoperabilidad de los contenidos no sólo entre los archivos, sino entre bibliotecas, museos e instituciones culturales en espacios web.

En la mayoría de los modelos, excepto en el modelo finlandés, se han definido propiedades para describir los objetos archivísticos. Los atributos se corresponden con las categorías de identificación, contexto, contenidos, estructura, gestión, características formales y notas de carácter general. Estas propiedades se pueden actualizar de acuerdo a la práctica descriptiva de cada país. Un ejemplo aplicado de atributos para la entidad documento en el MCDA RiC-CM se registra en la tabla 2, donde las categorías RiCP1/P21 de identificación, de contenidos, de representación, estructurales, y otras características sobre su gestión y uso son factibles de reconocer.

Tabla 2. Atributos para Documento en RiC-CM

\begin{tabular}{|c|l|c|}
\hline \multicolumn{3}{|c|}{1 MODELO CONCEPTUAL INTERNACIONAL } \\
\hline \multicolumn{2}{|c|}{$2 \quad$ ATRIBUTOS } \\
\hline$\#$ & \multicolumn{1}{|c|}{ PARA TODAS LA ENTIDADES } & \\
\hline RiC-P1 & Identificador global (para todas las entidades) & Identificación \\
\hline RiC-P2 & Identificador local (para todas las entidades) & \\
\hline RiC-P3 & Nombre(para todas las entidades) & \\
\hline RiC-P4 & Nota general(para todas las entidades) & Contenido \\
\hline & \multicolumn{1}{|c|}{ ENTIDAD DOCUMENTO } \\
\hline RiC-P5 & Nota de autenticidad e integridad & \\
\hline RiC-P6 & Tipo de Contenido & \\
\hline
\end{tabular}




\begin{tabular}{|l|l|l|}
\hline RiC-P7 & Extensión de contenido & \\
\hline RiC-P8 & Calidad de información & \\
\hline RiC-P9 & Alcance y contenido & Representación \\
\hline RiC-P10 & Formato de codificación & \\
\hline RiC-P11 & Lengua/Escritura & \\
\hline RiC-P12 & Tipo de medio o equipo para reproducir & Continente \\
\hline RiC-P13 & Requisitos técnicos de producción & \\
\hline RiC-P14 & Material físico en el que se representa la información & \\
\hline RiC-P15 & Extensión física y lógica & Gestión y uso \\
\hline RiC-P16 & Nota sobre características físicas & \\
\hline RiC-P17 & Clasificación & \\
\hline RiC-P18 & Condiciones de acceso & \\
\hline RiC-P19 & Condiciones de uso & \\
\hline RiC-P20 & Historia & \\
\hline RiC-P21 & Estado del documento & \\
\hline
\end{tabular}

Fuente: Elaboración propia

En este ejemplo se visualizan variaciones importantes en los atributos relacionadas con los nuevos propósitos de la descripción en cuanto a interoperabilidad, reutilización, publicación de datos abiertos y preservación; los metadatos nota de autenticidad e integridad, calidad de información, formato de codificación o estado del documento (copia, borrador, original), entre otros, así lo evidencian.

La ejemplificación de las relaciones entre las entidades es otro aspecto determinante en un modelo conceptual. En todos los MCDA se presentan relaciones contextuales, donde es reflejado el entorno de creación y uso de los documentos. La determinación de una amplia variedad de relaciones garantiza la interrelación de los contenidos de los documentos con los diversos contextos en los que fueron producidos. Igualmente, el establecimiento de las redes relacionales facilita la exposición gráfica de los contenidos descriptivos en el entorno tecnológico y a posteriori garantizará el incremento de la capacidad de búsqueda de los sistemas, así como la recuperación y el uso de la información. La descripción ya no se concibe en jerarquías sino en redes de conocimiento interconectadas con disímiles recursos digitales.

\section{Ciclo de vida de la Curaduría Digital}

De acuerdo con el Digital Curation Center, DCC (2017), la Curaduría Digital incluye el almacenamiento de datos, información y documentos y su conservación. Tal y como se ha señalado, para su logro precisa de la descripción, así como de una planificación de la preservación. Ante todo, requiere de un análisis previo conceptual sobre el OD, sobre su información asignada, así como sobre las necesidades de uso del recurso informativo digital. Esto es, se debe de responder a interrogantes tales como qué se almacena (formato, tipología, extensión, propiedades, etc.); qué se conserva (normas básicas y metodología a emplear incluidos los metadatos); para quién y cuáles son sus futuros usos (comunidades de usuarios y planes de acceso y de explotación del OD) y, finalmente, durante cuánto tiempo ha de almacenarse y/o conservarse (estrategias de almacenamiento, de valoración y de conservación del recurso). De igual modo, han de 
delimitarse los requisitos legales vinculados a acceso, restricción y propiedad industrial (intelectual u otras), así como los roles y responsabilidades sobre ambas acciones. En el ciclo de vida de la $\mathrm{CD}$, una vez "conceptualizado" el recurso digital, pueden observarse las siguientes acciones: creación/recepción, acceso y uso en aplicaciones informáticas, selección (previa valoración), ingesta o captura en repositorios, actuación preservadora, almacenamiento, acceso y reutilización y, en su caso, transformación. Esta última, atañe tanto a la estrategia de conservación del OD (migración, etc.), como a la reutilización. Los procesos de CD, según Santos (2014), garantizan a los usuarios el acceso, uso y reutilización de información digital en repositorios a corto, medio y largo plazo; de esta forma, la conservación y el acceso a los recursos digitales se convierten en la espina dorsal de este ciclo. De facto, requiere una gestión proactiva que se inicia en el análisis contextual del recurso ante su creación/recepción.

Con este modelo se pretende asegurar la autenticidad, confiabilidad, integridad y usabilidad de los objetos digitales; para tal fin, se platean otras acciones permanentes a lo largo del ciclo de vida relacionadas con la descripción, la representación, la valoración o la validación de información. La calidad de la información está presente en buena parte de las etapas de la $\mathrm{CD}$, tanto en la creación, como en la ingesta y en la disponibilidad del recurso digital de información.

El modelo del ciclo de vida de la Curaduría Digital contiene varias etapas que se visualizan en un diagrama elaborado por el DCC y que se muestra en la siguiente figura.

Figura 1. Ciclo de Vida de la Curaduría Digital

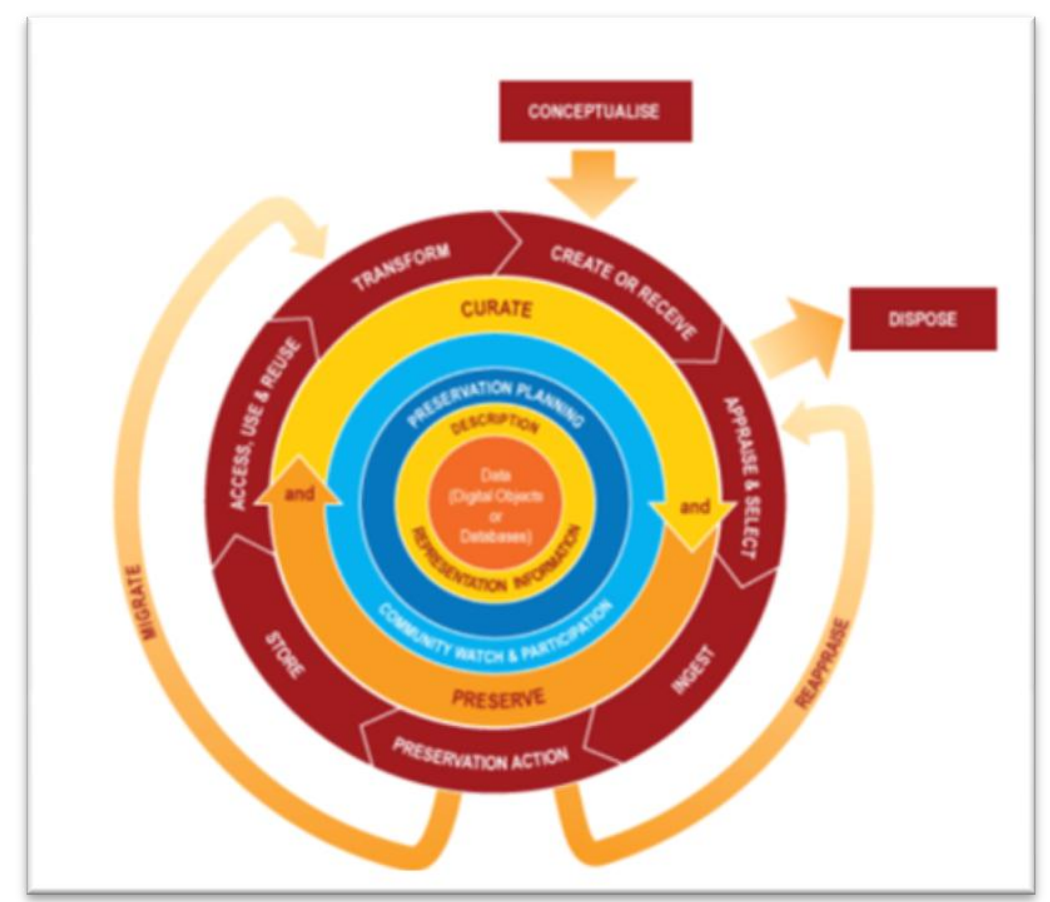

Fuente: Tyler Walters, Virginia Tech, and Katherine Skinner. New Roles for New Times: Digital Curation for Preservation.

La CD vincula la descripción a la etapa de creación/recepción del recurso digital mediante la asignación de metadatos de archivo, incluidos los de almacenamiento y conservación. Asimismo, el acceso, uso y reutilización de los documentos se efectuará 
siempre que exista una adecuada representación de la información. Los atributos de los estándares favorecen su cumplimiento, en cuanto a identificación, descripción de los contenidos, contextualización, normalización de los datos, integridad, disponibilidad, etc. Para una eficiente CD se deben concebir planes de acción para todas las etapas del ciclo y así evitar problemas de preservación, pudiendo diseminar los recursos digitales en períodos variables, sea a corto, sea a largo plazo.

Si pensamos en el modelo del record continuum, definido por el australiano Frank Upward, en él se explican la evolución, cambios y usos del documento de archivo durante su ciclo de vida continuado en pro de la gestión documental o el mantenimiento de la memoria. Se reconocen cuatro dimensiones concernidas con la creación, la captura, organización y diseminación; resulta sencillo comparar estas dimensiones con las establecidas, tanto en el modelo de CD (conceptualización, creación-recepcióningesta, valoración-disposición, estrategias conservadoras, almacenamiento, acceso, uso y reutilización), como en aquellas categorías de requisitos funcionales para un sistema de gestión de documentos acordadas en la norma UNE-ISO 16175-2 (Aenor, 2012), donde son identificables las de creación-captura, mantenimiento y difusión de los documentos. En todas ellas, la consignación de metadatos es esencial, tanto para la identificación, descripción de contenidos y contextualización, como para la organización funcional y de almacenamiento, así como para la recuperación y representación de la información.

A partir de este diligente análisis, se puede decir que existe una relación estrecha entre CD y el modelo australiano (donde los puntales de creación/captura, mantenimiento y diseminación se reiteran), especialmente con los procesos continuos para el mantenimiento de documentos de archivo. A su vez, queda reflejado, incluso en los requisitos tecnológicos necesarios, tal y como se refleja en la figura siguiente.

Figura 2. Comparativa de fases reconocidas en la CD, modelo RC e ISO tecnológico de requisitos.

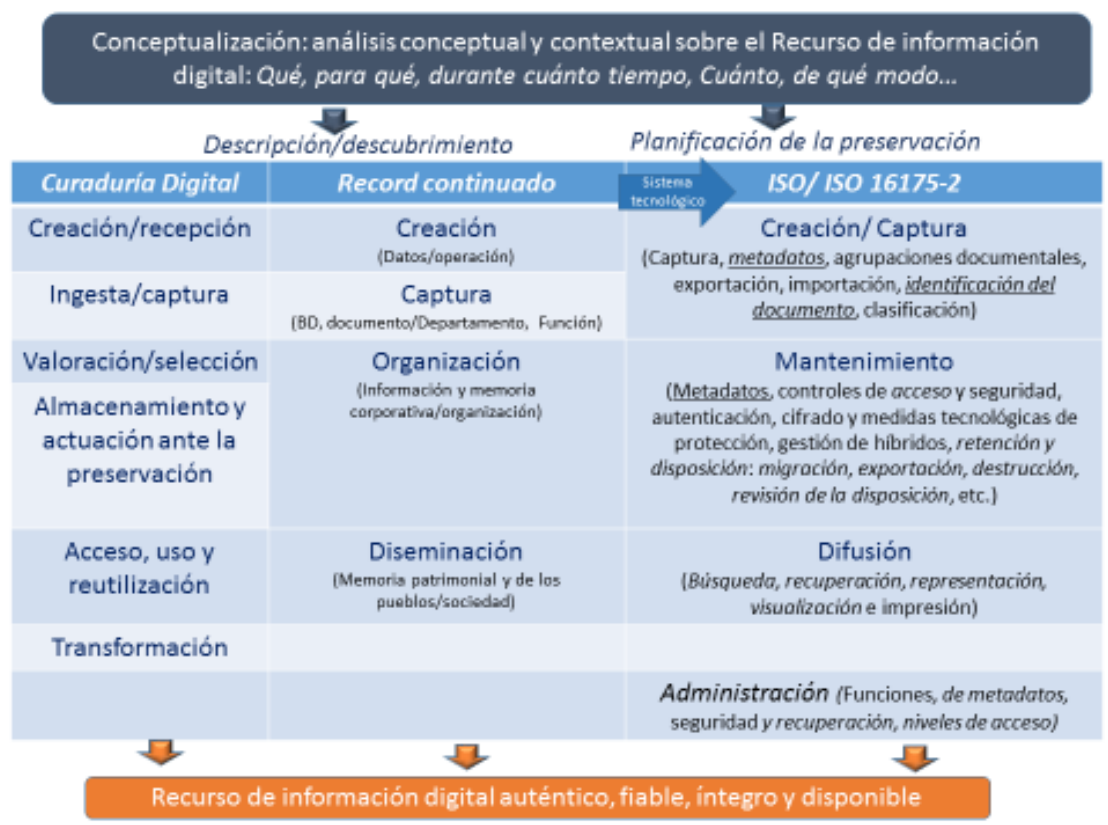

Fuente: Elaboración propia 
En este escenario actual de convergencias y complejidades es necesario tener en cuenta para las estrategias de las etapas del ciclo de vida de la $\mathrm{CD}$, el conjunto de los requisitos normativos y teóricos instaurados para procesos archivísticos. Los entornos electrónicos exigen demostraciones de relaciones e interoperabilidad de la información ante sus diversos sistemas de gestión; por tanto, las normas técnicas, los modelos conceptuales, los desarrollos ontológicos -elaborados en el seno de ISO, del ICA, así como de otros organismos nacionales-, aglutinados demuestran gran utilidad. Esto es: las normas para representar y recuperar contenidos, para facilitar la continuidad y preservación de los recursos digitales, así como para construir y certificar sistemas de gestión de calidad, todas ellas ayudarán a la aplicación con éxito de este modelo de Curaduría Digital.

\section{Descripción, acceso y curaduría digital: relaciones posibles}

En la descripción archivística del s. XXI, se reconocen tres propósitos bien definidos: gestión documental; preservación de documentos y reutilización de datos (ICA; 2016). La descripción se adapta a los nuevos tiempos y a través de la representación de contenidos y contextos se contribuye con la ejecución de estas funciones. Los modelos conceptuales incluyen como hilo conductor estos nuevos propósitos.

Esta relación se visibiliza con el valor de evidencia. Así, cuando se describen entidades archivísticas con los atributos definidos en los MCDA, se viabiliza el mantenimiento de la autenticidad e integridad de los documentos. En RiC-CM se expone que "documentar el contexto describiéndolo es esencial para la conservación de los registros" (ICA, 2016). Por consiguiente, en este medio, es importante resaltar que los metadatos descriptivos son determinantes para la conservación de la memoria mundial a largo plazo. (LLanes y Moro, 2017). Al respecto, cabe reconocer que la identificación del recurso digital, su descripción tecnológica, el aseguramiento de su integridad y autenticidad, el control de los derechos legales, entre otros, forman parte de ese paquete descriptivo, a la par que proporcionan cabida a una diversidad de metadatos considerados igualmente necesarios para su preservación.

Si observamos el diagrama del ciclo de vida de la curaduría digital, se puede percibir que la descripción está puntualizada como un proceso central en el modelo, o lo que es lo mismo, es una actividad de ejecución permanente durante todo el ciclo de vida del objeto digital. Por ende, se hace necesario incluir, en las estrategias de la CD, las bases de los MCDA, pues son estos modelos los que convierten a la descripción en un proceso eficaz y adaptado a las transformaciones digitales facilitando mediante atributos y relaciones entre entidades, el uso de la información, su reutilización, la preservación y la interoperabilidad entre sistemas. De facto, crear, recibir y capturar exige el despliegue de metadatos de identificación, descripción, contextuales, tecnológicos y de conservación.

El proceso de descripción está directamente vinculado con el acceso del patrimonio archivístico; si se piensa en términos de proporcionalidad, a mayor calidad-eficiencia en la descripción de un recurso, mayor calidad-eficiencia en su búsqueda, recuperación y reutilización. En suma, la normalización de este proceso debe contemplarse como el camino correcto para aportar valor al ciclo de vida de la CD. Para ello, necesita el empleo de modelos conceptuales descriptivos y de ontologías. 
En la siguiente tabla se especifican las relaciones de los atributos de RiC-CM en relación con el estándar de diseño OAIS y el estándar PREMIS, específico para facilitar la gestión de metadatos de preservación. Se observa como la información que se aconseja en el estándar OAIS, tanto en lo descriptivo como en lo referente a preservación con sus categorías de metadatos (tres columnas primeras), puede ser correspondida desde PREMIS, especialmente para la Preservación, debido a que la norma se focaliza en ella ( $5^{\mathrm{a}}$ columna), no primando la descripción estática del recurso informativo digital. Destacan dos entidades: Objeto y Derechos. Se detallan en ambas las unidades semánticas que implican mayor grado de incidencia. Finalmente, en la columna $4^{\mathrm{a}}$ se consideraron los atributos para la entidad Documento de RiC-MC. Estos son distribuidos bajo las taxonomías siguientes: continente, representación, contenido, gestión y uso e identificador para todas las entidades.

Tabla 3. Visualización de relaciones entre la descripción y la preservación basada en los estándares.

\begin{tabular}{|c|c|c|c|c|}
\hline $\begin{array}{c}\text { OAIS } \\
\text { (Información) }\end{array}$ & $\begin{array}{c}\text { OAIS } \\
\text { (Categorías } \\
\text { Metadatos } \\
\text { Preservación) }\end{array}$ & $\begin{array}{c}\text { OAIS } \\
\text { (Categorías } \\
\text { Alcance) }\end{array}$ & $\begin{array}{c}\text { RiC-CM } \\
\text { (Atributos } \\
\text { Entidad } \\
\text { Documento) }\end{array}$ & $\begin{array}{c}\text { PREMIS } \\
\text { (Diccionario de } \\
\text { Datos) }\end{array}$ \\
\hline $\begin{array}{l}\text { Empaqueta- } \\
\text { miento }\end{array}$ & & $\begin{array}{l}\text { Permite la } \\
\text { identificación del } \\
\text { fichero con sus } \\
\text { metadatos. }\end{array}$ & $\begin{array}{l}\text { Continente de la } \\
\text { información: } \\
\text { RiC-P15/RiC- } \\
\text { P16. Extensión } \\
\text { física y lógica; } \\
\text { notas sobre } \\
\text { características } \\
\text { físicas. }\end{array}$ & $\begin{array}{l}\text { Entidad objeto. } \\
\text { Tamaño y } \\
\text { extensión. } \\
\text { Almacenamiento. }\end{array}$ \\
\hline Descriptiva & estática & $\begin{array}{l}\text { Descripción para } \\
\text { la recuperación } \\
\text { (se arrastra en } \\
\text { preservación, } \\
\text { localización) }\end{array}$ & $\begin{array}{l}\text { Representación: } \\
\text { RiC-P10/RiC- } \\
\text { P14. Formato; } \\
\text { escritura o } \\
\text { lengua; tipo de } \\
\text { equipo para } \\
\text { reproducción; } \\
\text { Requisitos } \\
\text { técnicos de } \\
\text { reproducción; } \\
\text { Material físico } \\
\text { en que se } \\
\text { presenta; } \\
\text { Extensión física } \\
\text { y lógica }\end{array}$ & $\begin{array}{l}\text { PREMIS no se } \\
\text { centra en } \\
\text { metadatos } \\
\text { descriptivos }\end{array}$ \\
\hline Contenido & & $\begin{array}{l}\text { Informa sobre el } \\
\text { contenido }\end{array}$ & $\begin{array}{l}\text { Contenido: } \\
\text { RiC-P6/RiC- } \\
\text { P9. Tipo y } \\
\text { extensión del } \\
\text { contenido. }\end{array}$ & $\begin{array}{l}\text { Entidad Objeto. } \\
\text { Categoría y } \\
\text { características } \\
\text { del objeto. } \\
\text { Propiedades }\end{array}$ \\
\hline
\end{tabular}




\begin{tabular}{|c|c|c|c|c|}
\hline $\begin{array}{c}\text { OAIS } \\
\text { (Información) }\end{array}$ & $\begin{array}{c}\text { OAIS } \\
\text { (Categorías } \\
\text { Metadatos } \\
\text { Preservación) }\end{array}$ & $\begin{array}{c}\text { OAIS } \\
\text { (Categorías } \\
\text { Alcance) }\end{array}$ & $\begin{array}{c}\text { RiC-CM } \\
\text { (Atributos } \\
\text { Entidad } \\
\text { Documento) }\end{array}$ & $\begin{array}{c}\text { PREMIS } \\
\text { (Diccionario de } \\
\text { Datos) }\end{array}$ \\
\hline & & & $\begin{array}{l}\text { Calidad de la } \\
\text { información; } \\
\text { alcance y } \\
\text { contenido. }\end{array}$ & $\begin{array}{l}\text { significativas. } \\
\text { Características } \\
\text { del objeto: } \\
\text { tamaño y } \\
\text { extensión. }\end{array}$ \\
\hline \multirow[t]{3}{*}{ Preservación } & $\begin{array}{l}\text { dinámica } \\
\text { Localización/ } \\
\text { descripción }\end{array}$ & $\begin{array}{l}\text { Identificación del } \\
\text { recurso de } \\
\text { información } \\
\text { digital y } \\
\text { descripción del } \\
\text { mismo }\end{array}$ & $\begin{array}{l}\underline{\text { Identificador }} \\
\text { para todas las } \\
\text { entidades. RiC- } \\
\text { P1/RiC-P4. } \\
\text { Identificadores } \\
\text { globales, } \\
\text { locales, nombre } \\
\text { y nota general. } \\
\text { Acompañan } \\
\text { atributos } \\
\text { descriptivos y } \\
\text { de } \\
\text { representación. }\end{array}$ & $\begin{array}{l}\text { Entidad objeto. } \\
\text { Identificador del } \\
\text { objeto. Nombre } \\
\text { original. } \\
\text { Identificadores de } \\
\text { la entidad } \\
\text { intelectual. }\end{array}$ \\
\hline & Procedencia & $\begin{array}{l}\text { Historial } \\
\text { contenido, } \\
\text { evolución OD y } \\
\text { de su } \\
\text { conservación. }\end{array}$ & $\begin{array}{l}\text { Gestión y uso. } \\
\text { RiC-P20. } \\
\text { Historia. } \\
\text { Acompañan } \\
\text { atributos de } \\
\text { contenido. }\end{array}$ & 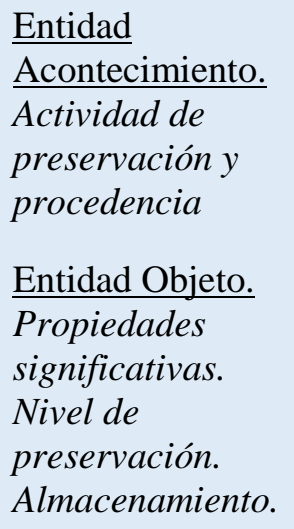 \\
\hline & Evidencia & $\begin{array}{l}\text { Autenticidad e } \\
\text { integridad: } \\
\text { firmas, marcas de } \\
\text { agua, sellado de } \\
\text { tiempo, } \\
\text { encriptación, } \\
\text { documentación } \\
\text { de autenticidad }\end{array}$ & $\begin{array}{l}\text { Contenido: } \\
\text { RiC-P5. Nota } \\
\text { de autenticidad } \\
\text { e integridad. } \\
\text { RiC-P8 Calidad } \\
\text { de la } \\
\text { información }\end{array}$ & $\begin{array}{l}\text { Entidad Objeto. } \\
\text { Características } \\
\text { del objeto: fijeza. } \\
\text { Información } \\
\text { sobre firma. } \\
\text { Inhibidores. }\end{array}$ \\
\hline
\end{tabular}




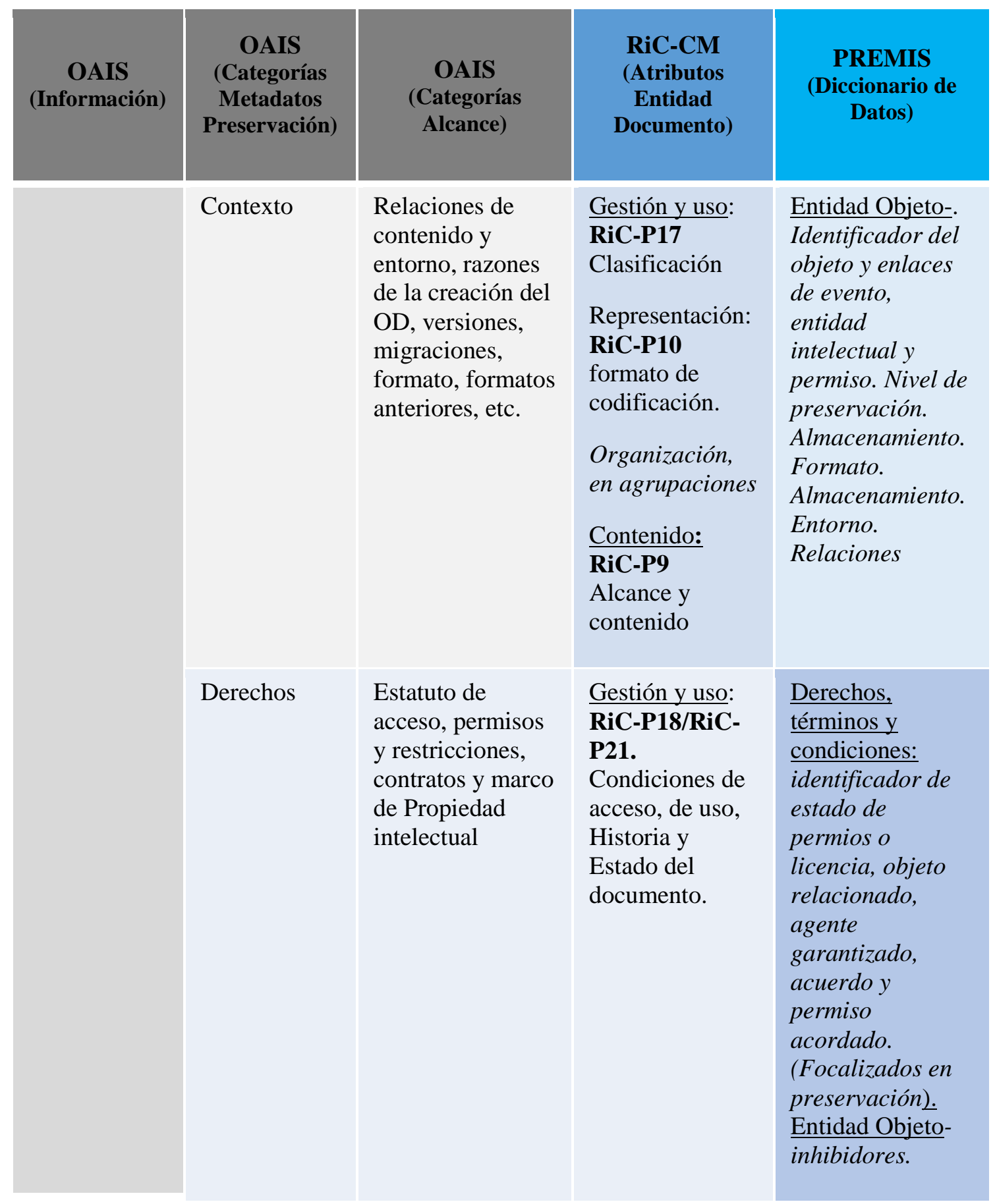

Fuente: Elaboración propia.

La tabla es una muestra de concordancias entre las relaciones que han de establecerse entre ambas funciones, la descriptiva y la preservadora, con un objetivo prioritario, el de disponer el recurso de información digital en el tiempo. 


\section{Conclusiones}

Las actuales configuraciones de modelos y procesos informacionales en entornos digitales traen consigo nuevas expectativas para la difusión, preservación e integración de datos archivísticos. Con la aplicación de los modelos conceptuales dentro del ciclo de vida de la Curaduría Digital, los archivos, en general, se beneficiarán de las ventajas de las tecnologías en lo concerniente a mantenimiento, representación, interoperabilidad, reutilización de datos, servicios basados en acceso abierto y conservación. A los archivos, en calidad de mediadores entre productores y usuarios necesitados de información que han de poner en valor los datos, la información y los documentos, o/y en calidad de centros de investigación y de cultura, les corresponde ofrecer acceso, inteligibilidad de la información, facilitar su uso y, en su caso, su reutilización, además de conservar los recursos digitales. Esta doble finalidad favorecerá su función mediadora y de puesta en valor de los datos, información y documentos, así como aquella de mantenimiento de la memoria de las naciones a largo plazo.

\section{Referencias}

AENOR. UNE-ISO 16175-2 (2012). Información y Documentación. Principios y requisitos funcionales para documentos en entornos de oficina electrónica. Parte 2Directrices y Requisitos funcionales para sistemas que gestionan documentos electrónicos. Madrid.

AENOR. UNE-ISO 30300 (2011). Información y Documentación. Gestión de sistemas para los documentos. Fundamentos y Vocabulario. Madrid.

AENOR. UNE-ISO 14721 (2015). Sistemas de transferencia de datos e información espaciales. Sistema abierto de información de archivo (OAIS). Modelo de referencia. Madrid.

Diccionario de datos PREMIS. De metadatos de preservación. Versión 2.0. (2003) Biblioteca Nacional España. Recuperado de: http://www.loc.gov/standards/premis/PREMIS_es.pdf

DCC. Digital Curation Centre. (2017). What is Digital Curation? Recuperado de: http://www.dcc.ac.uk/digital-curation/what-digital-curation

DTIC. Dirección de Tecnologías de la Información y Comunicación (2016). Guía de aplicación de la norma técnica de interoperabilidad de políticas de gestión de documentos electrónicos. Madrid: Ministerio de Hacienda y Administración Pública. $2^{a}$ edición. Recuperado de: http://adminsistracionelectrónica.gob.es

ICA (2016). Records in contexts: A conceptual model for archival description. Recuperado de: http://www.ica.org/sites/default/files/RiC-CM-0.1.pdf

Llanes, D., Moro, M. (2017). Records in contexts: Un nuevo modelo para la representación de la información archivística en el entorno de la web semántica. $E l$ profesional de la información, v. 26 (n. 3), pp. 525-533.

Santos, T. N. C.( 2014). Curadoria digital: o conceito no período de 2000 a 2013. 
165 f. Tesis de Máster. Universidad de Brasília, Brasil. Recuperado de: http://repositorio.unb.br/bitstream/10482/17324/1/2014_ThayseNataliaCantanhedeSan tos.pdf.

Walters T., Tech V., Skinner K. (2011). New Roles for New Times: Digital Curation for Preservation . Washington: Association of Research Libraries. 